\title{
Baryon asymmetry and dark matter from soft leptogenesis
}

\author{
Heidi Kuismanen and Iiro Vilja \\ Department of Physics and Astronomy, University of Turku, 20014 Turku, Finland
}

August 13, 2018

\begin{abstract}
The framework for soft leptogenesis minimally extended with a DM sector is studied. A heavy singlet neutrino superfield acts as the source for (s)lepton asymmetry and by coupling to the singlet DM superfield it produces a DM particle density through decays. The nature of DM generated is twofold depending on whether the Yukawa and DM couplings are either small or large. With sufficiently small Yukawa and DM couplings DM annihilations into MSSM particles are slow and as a consequence all DM particles form the DM component. The solutions to Boltzmann equations are given and the dependence between the DM masses and coupling are presented in this weak coupling regime. Also, the behavior of the efficiency of producing asymmetric DM is determined with weak couplings. We note that a different outcome arises if the couplings are larger because then the ADM component is dominant due to the effectiveness of DM decays into the MSSM sector.
\end{abstract}

\section{Introduction}

Our current understanding of the universe is that around $5 \%$ of the energy density belongs to ordinary baryonic matter while the rest stems from dark matter (DM) and dark energy with proportions $22 \%$ and $73 \%$, respectively [1]. The existence of all three components is more or less a mystery and points towards physics beyond the Standard Model (SM). The fact that there seems to be almost exclusively matter and no antimatter in the universe suggests there is matter-antimatter asymmetry. Since the SM cannot accommodate sufficient baryogenesis on its own, baryogenesis mechanisms have been constructed in various Grand Unified Theory 
(GUT) scenarios. Alongside GUT models, baryogenesis via leptogenesis 2] has been studied extensively over the last twenty years or so.

As for DM, a viable candidate arises in the Minimal Supersymmetric Standard Model (MSSM), namely the Lightest Supersymmetric Particle (LSP) [3, 4]. Usually, the lightest neutralino (i.e. a combination of the superpartners of the neutral gauge bosons and higgs sparticles), or in supergravity theories the gravitino, assumes the role of the LSP, and the success of the LSP in producing the correct DM abundance depends on the adjustment of parameters within the MSSM. However, since the DM and baryonic energy densities are close to each other, $\Omega_{\mathrm{DM}} / \Omega_{\mathrm{b}} \sim 5$, it is natural to assume that $\mathrm{DM}$ and baryogenesis are somehow related. The connection between baryogenesis and DM has also been widely explored [5]. Moreover, the possibility of the DM energy density being the product of an asymmetry has led to the concept of asymmetric DM (ADM) where DM and DM antiparticles are distinct from another and produce an asymmetry analogous to baryons. Note, however, that as ADM scenarios incorporate MSSM, also LSP is always included in the ADM models but is not necessarily good candidate for DM.

Over the years various realizations of leptogenesis have been devised [6]-[28]. They are usually motivated by $\mathrm{SO}(10)$ scenarios where $B-L$ is a gauge symmetry, and it is natural to have a heavy right-handed SM singlet neutrino that can also be responsible for light neutrino masses via the seesaw mechanism [24, 25]. Adding a heavy singlet neutrino superfield into the Minimal Supersymmetric SM (MSSM) gives rise to soft leptogenesis where the soft breaking of supersymmetry allows the scalar superpartner of the heavy neutrino to generate the (s)lepton asymmetry [26, 27]. In contrast to non-SUSY models, only one family of heavy singlets is required.

Furthermore, various scenarios incorporating leptogenesis and DM have been introduced [29]-37]. In this article, we investigate a model that encompasses soft leptogenesis and DM generation from the same heavy singlet superfield. Previously a model with $S U(2)_{L}$ triplet DM and supersymmetric leptogenesis has been studied [38]. Our model is a minimal extension to the Minimal Supersymmetric Standard Model (MSSM) and it consists of MSSM augmented with a single SM singlet DM superfield and a heavy singlet neutrino superfield that enables soft leptogenesis after soft SUSY breaking. In section 2 we introduce our model and present the $\mathrm{CP}$ violation parameters, in section 3 we solve the Boltzmann equations and the resulting baryon and DM abundances, and in section 4 we present our conclusions. 


\section{The model}

Models incorporating leptogenesis and DM generation have the potential to produce the observed matter and DM energy densities and offer a possible explanation as to why the abundances are similar in magnitude. So far the MSSM has been considered as one of the most appealing extension to the SM, and with the addition of a heavy singlet (s)neutrino, soft leptogenesis can arise. To account for DM, the soft leptogenesis framework can be extended with an additional DM sector. We have augmented the MSSM with a singlet superfield consisting of a heavy neutrino and sneutrino and a singlet DM superfield. The heavy (s)neutrino couples to the MSSM (s)leptons, Higgses and higgsinos, which gives rise to the soft leptogenesis scenario. The DM sector couples to the heavy (s)neutrino as well and this provides additional decay channels for the singlet (s)neutrino and a source for DM production through these decays. The superpotential is

$$
W=W_{\mathrm{MSSM}}+y_{\alpha} N L_{\alpha} H_{u}+\frac{1}{2} M N N+m_{\Phi} \Phi \Phi^{c}+\frac{1}{2} \lambda N \Phi \Phi .
$$

The discrete symmetry $Z_{2}$ imposed on $\Phi$ forbids terms $\sim \Phi^{3}$ and $\sim N N \Phi$. After adding soft supersymmetry breaking terms we get the part of the Lagrangian that includes the soft leptogenesis and darkgenesis interactions

$$
\begin{aligned}
\mathcal{L}= & \lambda \phi N \chi+y_{\alpha} H_{u} L_{\alpha} N+y_{\alpha} \widetilde{L}_{\alpha} \widetilde{H}_{u} N+\text { h.c. } \\
& +\widetilde{N}_{+}\left[\frac{1}{\sqrt{2}} \lambda e^{-i \theta_{b} / 2} \chi \chi+\frac{1}{\sqrt{2}} y_{\alpha} e^{-i \theta_{b} / 2} L_{\alpha} \widetilde{H}_{u}+\frac{1}{2 \sqrt{2}} \lambda M^{*} e^{i \theta_{b} / 2} \phi^{2}\right. \\
& \left.+\frac{1}{\sqrt{2}} y_{\alpha} M^{*} e^{i \theta_{b} / 2} \widetilde{L}_{\alpha} H_{u}+\frac{1}{2} c_{1} e^{-i \theta_{b} / 2} \phi^{2}+\frac{1}{2} c_{2} e^{-i \theta_{b} / 2} \widetilde{L}_{\alpha} H_{u}+\text { h.c. }\right] \\
& +\widetilde{N}_{-}\left[\frac{i}{\sqrt{2}} \lambda e^{-i \theta_{b} / 2} \chi \chi+\frac{i}{\sqrt{2}} y_{\alpha} e^{-i \theta_{b} / 2} L_{\alpha} \widetilde{H}_{u}-\frac{i}{2 \sqrt{2}} \lambda M^{*} e^{i \theta_{b} / 2} \phi^{2}\right. \\
& \left.-\frac{i}{\sqrt{2}} y_{\alpha} M^{*} e^{i \theta_{b} / 2} \widetilde{L}_{\alpha} H_{u}+\frac{i}{\sqrt{2}} c_{1} e^{-i \theta_{b} / 2} \phi^{2}+\frac{i}{\sqrt{2}} c_{2} e^{-i \theta_{b} / 2} \widetilde{L}_{\alpha} H_{u}+\text { h.c. }\right]
\end{aligned}
$$

where the sneutrino mass eigenstates are

$$
\begin{aligned}
& \widetilde{N}_{+}=\frac{1}{\sqrt{2}}\left(e^{i \theta_{b} / 2} \widetilde{N}+e^{-i \theta_{b} / 2} \widetilde{N}^{*}\right) \\
& \widetilde{N}_{-}=\frac{1}{\sqrt{2} i}\left(e^{i \theta_{b} / 2} \widetilde{N}-e^{-i \theta_{b} / 2} \widetilde{N}^{*}\right) .
\end{aligned}
$$

The DM particles $\chi$ and $\phi$ represent the DM fermion and scalar, respectively.

The lepton and DM asymmetries arise from the self-energy loop diagrams shown in Figs. $1(\mathrm{a})\left[1(\mathrm{f})\right.$. While the process $\tilde{N}_{ \pm} \rightarrow \chi \chi^{c}$ does not produce a DM asymmetry, i.e. an asymmetry 
between $\chi$ and $\chi^{c}$, the decays $\widetilde{N}_{ \pm} \rightarrow \phi \phi$ and $\widetilde{N}_{ \pm} \rightarrow \phi^{*} \phi^{*}$ can create an asymmetry between $\phi$ and $\phi^{*}$. This is the source for possible ADM in our model and the asymmetric component consists of scalar DM. The CP violation parameter for (s)lepton production is defined as

$$
\varepsilon_{L}=\frac{\sum_{f}\left[\Gamma\left(\widetilde{N}_{+} \rightarrow f\right)-\Gamma\left(\widetilde{N}_{+} \rightarrow \bar{f}\right)+\Gamma\left(\widetilde{N}_{-} \rightarrow f\right)-\Gamma\left(\widetilde{N}_{-} \rightarrow \bar{f}\right)\right]}{\sum_{f}\left[\Gamma\left(\widetilde{N}_{+} \rightarrow f\right)+\Gamma\left(\widetilde{N}_{+} \rightarrow \bar{f}\right)+\Gamma\left(\widetilde{N}_{-} \rightarrow f\right)+\Gamma\left(\widetilde{N}_{-} \rightarrow \bar{f}\right)\right]}
$$

where $f$ stands for the fermionic $\left(l_{\alpha} \widetilde{H}_{u}^{c}\right)$ and bosonic $\left(\tilde{l}_{\alpha} H_{u}\right)$ final states. The DM asymmetry is defined in a similar manner:

$$
\varepsilon_{\mathrm{DM}}=\frac{\Gamma\left(\widetilde{N}_{+} \rightarrow \phi \phi\right)-\Gamma\left(\widetilde{N}_{+} \rightarrow \phi^{*} \phi^{*}\right)+\Gamma\left(\tilde{N}_{-} \rightarrow \phi \phi\right)-\Gamma\left(\widetilde{N}_{-} \rightarrow \phi^{*} \phi^{*}\right)}{\Gamma\left(\widetilde{N}_{+} \rightarrow \phi \phi\right)+\Gamma\left(\widetilde{N}_{+} \rightarrow \phi^{*} \phi^{*}\right)+\Gamma\left(\widetilde{N}_{-} \rightarrow \phi \phi\right)+\Gamma\left(\widetilde{N}_{-} \rightarrow \phi^{*} \phi^{*}\right)}
$$


The temperature-dependent $\mathrm{CP}$ violation parameter for the (s)leptons is

$$
\begin{aligned}
& \varepsilon_{L}(T)=\left[-\frac{4}{16 \pi} \frac{M_{\widetilde{N}_{+}}^{2}-M_{\widetilde{N}_{-}}^{2}}{\left(M_{\widetilde{N}_{+}}^{2}-M_{\widetilde{N}_{-}}^{2}\right)^{2}+\Pi_{--}^{2}} \frac{|M|}{v_{u}^{2}} m_{\mathrm{eff}}\right. \\
& \times\left(\frac{|M|^{2}}{2}-\frac{|A|^{2}}{2}\right)\left(2|A||M| \cos \left(\theta_{A}+\frac{\pi}{2}+\theta_{M}-\theta_{b}\right) \frac{|M|}{v_{u}^{2}} m_{\mathrm{eff}}\right. \\
& \left.+|\lambda||M|\left|c_{1}\right| \cos \left(-\theta_{\lambda}+\frac{\pi}{2}+\theta_{M}-\theta_{b}+\theta_{c_{1}}\right)\right) \frac{c_{B}}{16 \pi M_{\widetilde{N}_{+}}} \\
& -\frac{4}{16 \pi} \frac{M_{\widetilde{N}_{-}}^{2}-M_{\widetilde{N}_{+}}^{2}}{\left(M_{\widetilde{N}_{-}}^{2}-M_{\widetilde{N}_{+}}^{2}\right)^{2}+\Pi_{++}^{2}}\left(-\frac{|M|^{2}}{2}+\frac{|A|^{2}}{2}\right) \frac{|M|}{v_{u}^{2}} m_{\mathrm{eff}} \\
& \times\left(2|M||A| \cos \left(\theta_{M}+\theta_{A}-\theta_{b}+\frac{\pi}{2}\right) \frac{|M|}{v_{u}^{2}} m_{\mathrm{eff}}\right. \\
& \left.+\left|c_{1}\right||\lambda||M| \cos \left(\theta_{c_{1}}-\theta_{\lambda}+\theta_{M}-\theta_{b}+\frac{\pi}{2}\right)\right) \frac{c_{B}}{16 \pi M_{\widetilde{N}_{-}}} \\
& -\frac{4}{16 \pi} \frac{M_{\widetilde{N}_{-}}^{2}-M_{\widetilde{N}_{+}}^{2}}{\left(M_{\widetilde{N}_{-}}^{2}-M_{\widetilde{N}_{+}}^{2}\right)^{2}+\Pi_{++}^{2}} \frac{|M|}{v_{u}^{2}} \frac{m_{\mathrm{eff}}}{2}\left(2|M||A| \sin \left(-\theta_{M}-\theta_{A}+\theta_{b}\right) \frac{|M|}{v_{u}^{2}} m_{\mathrm{eff}}\right. \\
& \left.+\left|c_{1}\right||\lambda||M| \sin \left(\theta_{\lambda}-\theta_{c_{1}}-\theta_{M}+\theta_{b}\right)\right) \frac{c_{F}}{16 \pi} M_{\widetilde{N}_{-}} \\
& -\frac{4}{16 \pi} \frac{M_{\widetilde{N}_{+}}^{2}-M_{\widetilde{N}_{-}}^{2}}{\left(M_{\widetilde{N}_{+}}^{2}-M_{\widetilde{N}_{-}}^{2}\right)^{2}+\Pi_{--}^{2}} \frac{|M|}{v_{u}^{2}} \frac{m_{\mathrm{eff}}}{2}\left(2|A||M| \sin \left(\theta_{b}-\theta_{A}-\theta_{M}\right) \frac{|M|}{v_{u}^{2}} m_{\mathrm{eff}}\right. \\
& \left.\left.+\left|c_{1}\right||M||\lambda| \sin \left(\theta_{b}-\theta_{c_{1}}+\theta_{\lambda}-\theta_{M}\right)\right) M_{\widetilde{N}_{+}} \frac{c_{F}}{16 \pi}\right] \\
& \times\left[\left(|M|^{2}+|A|^{2}+2|M||A| \cos \left(\theta_{M}-\theta_{b}+\theta_{A}\right)\right) \frac{|M|}{v_{u}^{2}} m_{\mathrm{eff}} \frac{c_{B}}{8 \pi M_{\widetilde{N}_{+}}}\right. \\
& +\left(|M|^{2}+|A|^{2}-2|M||A| \cos \left(\theta_{M}-\theta_{b}+\theta_{A}\right)\right) \frac{|M|}{v_{u}^{2}} m_{\mathrm{eff}} \frac{c_{B}}{8 \pi M_{\widetilde{N}_{-}}} \\
& \left.+M_{\widetilde{N}_{-}} \frac{|M|}{v_{u}^{2}} m_{\mathrm{eff}} \frac{c_{F}}{8 \pi}+M_{\widetilde{N}_{+}} \frac{|M|}{v_{u}^{2}} m_{\mathrm{eff}} \frac{c_{F}}{8 \pi}\right]^{-1},
\end{aligned}
$$

where we see the influence of the DM sector couplings in the $\widetilde{N}_{ \pm}$self-energies and $\widetilde{N}_{ \pm} \rightarrow \widetilde{N}_{\mp}$ 
transitions. We have used $c_{2} \equiv A \sum_{\alpha} y_{\alpha}$ and $m_{\mathrm{eff}}=v_{u}^{2} \sum_{\alpha}\left|y_{\alpha}\right|^{2} / M$. The net $\phi$ asymmetry is

$$
\begin{aligned}
\varepsilon_{\mathrm{DM}}(T)= & {\left[-\frac{4}{16 \pi} \frac{M_{\widetilde{N}_{+}}^{2}-M_{\widetilde{N}_{-}}^{2}\left(M_{\widetilde{N}_{+}}^{2}-M_{\widetilde{N}_{-}}^{2}\right)^{2}+\Pi_{--}^{2}}{}\left(\frac{|M|^{2}|\lambda|^{2}}{8}-\frac{\left|c_{1}\right|^{2}}{2}\right) \frac{c_{\phi}}{16 \pi M_{\widetilde{N}_{+}}}\right.} \\
& \times\left(2|A||M| \cos \left(\theta_{A}+\frac{\pi}{2}+\theta_{M}-\theta_{b}\right) \frac{|M|}{v_{u}^{2}} m_{\mathrm{eff}}\right. \\
& \left.+\left|c_{1}\right||M| \lambda \sin \left(\theta_{b}-\theta_{c_{1}}+\theta_{\lambda}-\theta_{M}\right)\right) \\
& -\frac{4}{16 \pi} \frac{M_{\widetilde{N}_{-}}^{2}-M_{\widetilde{N}_{+}}^{2}}{\left(M_{\widetilde{N}_{-}}^{2}-M_{\widetilde{N}_{+}}^{2}\right)^{2}+\Pi_{++}^{2}}\left(\frac{\left|c_{1}\right|^{2}}{2}-\frac{|\lambda|^{2}|M|^{2}}{8}\right) \frac{c_{\phi}}{16 \pi M_{\widetilde{N}_{-}}} \\
& \left(2|M||A| \cos \left(\theta_{M}+\theta_{A}-\theta_{b}+\frac{\pi}{2}\right) \frac{|M|}{v_{u}^{2}} m_{\mathrm{eff}}\right. \\
& \left.\left.+\left|c_{1}\right||\lambda||M| \sin \left(\theta_{\lambda}+\theta_{b}-\theta_{c_{1}}-\theta_{M}\right)\right)\right] \\
& \times\left[\frac{c_{\phi}}{8 \pi M_{\widetilde{N}_{+}}}\left(\frac{\left|c_{1}\right|^{2}}{2}+\frac{|M|^{2}|\lambda|^{2}}{8}+\frac{1}{2}\left|c_{1}\right||M||\lambda| \cos \left(\theta_{c_{1}}-\theta_{b}+\theta_{M}-\theta_{\lambda}\right)\right)\right. \\
& \left.+\frac{c_{\phi}}{8 \pi M_{\widetilde{N}_{-}}}\left(\frac{|M|^{2}|\lambda|^{2}}{8}+\frac{\left|c_{1}\right|^{2}}{2}-\frac{1}{2}|M||\lambda|\left|c_{1}\right| \cos \left(\theta_{\lambda}-\theta_{M}+\theta_{b}-\theta_{c_{1}}\right)\right)\right]^{-1}
\end{aligned}
$$

with the factors 12

$$
\begin{aligned}
c_{F} & =\left(1-x_{l}-x_{\widetilde{H}}\right) \sqrt{\left(1+x_{l}-x_{\widetilde{H}}\right)^{2}-4 x_{l}}\left(1-f_{l}^{\mathrm{eq}}\right)\left(1-f_{\widetilde{H}}^{\mathrm{eq}}\right), \\
c_{B} & =\sqrt{\left(1+x_{H}-x_{\widetilde{l}}\right)^{2}-4 x_{H}}\left(1+f_{H}^{\mathrm{eq}}\right)\left(1+f_{\widetilde{l}}^{\mathrm{eq}}\right), \\
c_{\phi} & =\sqrt{1-4 x_{\phi}}\left(1+f_{\phi}^{\mathrm{eq}}\right)\left(1+f_{\phi}^{\mathrm{eq}}\right) \\
x_{a} & =\frac{m_{a}(T)^{2}}{M^{2}}, f_{a}^{\mathrm{eq}}=\frac{1}{e^{E_{a} / T} \pm 1},
\end{aligned}
$$

where the thermal masses are

$$
\begin{aligned}
m_{H}(T)^{2} & =T^{2}\left(\frac{3}{8} g_{2}^{2}+\frac{g_{Y}^{2}}{8}+\frac{3}{4} 4 \pi \alpha_{t}\right) \\
m_{\widetilde{H}}(T)^{2} & =\frac{1}{2} T^{2}\left(\frac{3}{8} g_{2}^{2}+\frac{g_{Y}^{2}}{8}+\frac{3}{4} 4 \pi \alpha_{t}\right) \\
m_{\widetilde{l}}(T)^{2} & =T^{2}\left(\frac{3}{8} g_{2}^{2}+\frac{g_{Y}^{2}}{8}\right) \\
m_{l}(T)^{2} & =\frac{1}{2} T^{2}\left(\frac{3}{8} g_{2}^{2}+\frac{g_{Y}^{2}}{8}\right) \\
m_{\phi}(T)^{2} & =m_{\Phi}^{2}+m_{\text {कsoft }}^{2}+\lambda^{2} T^{2}
\end{aligned}
$$


The phases $\theta_{M}$ and $\theta_{b}$ can be rotated away and are set to zero and so $M$ is real and we can replace $|M| \rightarrow M$. The factors $c_{F, B, \phi}$ reflect the production thresholds of MSSM particles and DM boson $\phi$ imposed by energy conservation. Since we normalize the DM asymmetry to the DM boson production, the factors $c_{\phi}$ cancel and consequently there is no temperature dependence in $\varepsilon_{\mathrm{DM}}$. We have also kept the soft terms at the vertices for completeness, usually these are neglected [27]. 


\section{Boltzmann equations}

We move on to solve the relic baryon and DM abundances this scenario generates. We assume the Yukawa couplings $m_{\text {eff }}$ and DM coupling $|\lambda|$ are sufficiently small and consider only processes with cross sections $\mathcal{O}\left(y^{2}\right)$ and $\mathcal{O}\left(\lambda^{2}\right)$. Due to this, DM annihilation processes are left out, which rules out ADM as the primary source of the DM energy density. Thus, we write the Boltzmann equations also for the total abundances of $\chi$ and $\phi$. The particle abundances are expressed in terms of the number densities normalized to entropy density $Y_{i} \equiv n_{i} / s$ and their evolution with changing $z \equiv M / T$ is to be determined. Our Boltzmann equations are

$$
\begin{aligned}
& \frac{d Y_{N}}{d z}=-\left(Y_{N}-Y_{N}^{\mathrm{eq}}\right)\left(D_{N}+D_{N \chi \phi}+4 S_{t}^{(0)}+4 S_{t}^{(1)}+4 S_{t}^{(2)}+2 S_{t}^{(3)}+4 S_{t}^{(4)}\right), \\
& \frac{d Y_{\widetilde{N}}}{d z}=-\left(Y_{\widetilde{N}}-2 Y_{\widetilde{N}}^{\mathrm{eq}}\right)\left[\frac{D_{\widetilde{N}}}{2}+D_{\widetilde{N}}^{(3)}+\frac{D_{\widetilde{N} \phi \phi}}{2}+\frac{D_{\widetilde{N} \chi \chi}}{2}+3 S_{22}\right. \\
& \left.+2\left(S_{t}^{(5)}+S_{t}^{(6)}+S_{t}^{(7)}+S_{t}^{(9)}\right)+S_{t}^{(8)}\right] \\
& \frac{d Y_{\Delta L_{\mathrm{tot}}}}{d z}=\varepsilon_{L}(T)\left(Y_{\widetilde{N}}-2 Y_{\widetilde{N}}^{\mathrm{eq}}\right) \frac{D_{\widetilde{N}}}{2} \\
& -\frac{Y_{\Delta L_{\mathrm{tot}}}}{Y_{l}^{\mathrm{eq}}}\left[\frac{D_{N}}{2} Y_{N}^{\mathrm{eq}}+\frac{D_{\widetilde{N}}}{2} Y_{\widetilde{N}}^{\mathrm{eq}}+Y_{\widetilde{N}}^{\mathrm{eq}} D_{\widetilde{N}}^{(3)}+Y_{\widetilde{N}}\left(S_{t}^{(5)}+\frac{S_{t}^{(8)}}{2}\right)\right. \\
& +Y_{N}\left(2 S_{t}^{(0)}+S_{t}^{(3)}\right)+2 Y_{N}^{\mathrm{eq}}\left(S_{t}^{(1)}+S_{t}^{(2)}+S_{t}^{(4)}\right) \\
& \left.+2 Y_{\widetilde{N}}^{\mathrm{eq}}\left(S_{t}^{(6)}+S_{t}^{(7)}+S_{t}^{(9)}\right)+S_{22}\left(2 Y_{\widetilde{N}}^{\mathrm{eq}}+\frac{Y_{\widetilde{N}}}{2}\right)\right] \\
& \frac{d Y_{\Delta \phi}}{d z}=\varepsilon_{\mathrm{DM}}\left(Y_{\widetilde{N}}-2 Y_{\widetilde{N}}^{\mathrm{eq}}\right) \frac{D_{\widetilde{N} \phi \phi}}{2}-\frac{Y_{\Delta \phi}}{Y_{\phi}^{\mathrm{eq}}}\left(\frac{D_{\widetilde{N} \phi \phi}+D_{\widetilde{N} \chi \chi}}{2} Y_{\widetilde{N}}^{\mathrm{eq}}+\frac{D_{N \chi \phi}}{2} Y_{N}^{\mathrm{eq}}\right), \\
& \frac{d Y_{\chi}}{d z}=-\left(\sqrt{1-\frac{4 m_{\chi}^{2}}{M^{2}}} D_{\widetilde{N} \chi \chi}+\sqrt{1+\frac{\left(m_{\chi}^{2}-m_{\phi}^{2}\right)^{2}}{M^{4}}-2 \frac{m_{\phi}^{2}+m_{\chi}^{2}}{M^{2}}} D_{N \chi \phi}\right) \\
& \times\left(Y_{\widetilde{N}}-2 Y_{\widetilde{N}}^{\mathrm{eq}}\right), \\
& \frac{d Y_{\phi}}{d z}=-\left(\sqrt{1-\frac{4 m_{\phi}^{2}}{M^{2}}} D_{\widetilde{N} \phi \phi}+\sqrt{1+\frac{\left(m_{\chi}^{2}-m_{\phi}^{2}\right)^{2}}{M^{4}}-2 \frac{m_{\phi}^{2}+m_{\chi}^{2}}{M^{2}}} D_{N \chi \phi}\right) \\
& \times\left(Y_{\widetilde{N}}-2 Y_{\widetilde{N}}^{\mathrm{eq}}\right) .
\end{aligned}
$$

with $Y_{\widetilde{N}}^{\text {eq }} \approx Y_{\widetilde{N}_{ \pm}}^{\text {eq }}$ and $Y_{\widetilde{N}} / 2 \approx Y_{\widetilde{N}_{ \pm}}$[39]. Recall that we take into account $\mathcal{O}\left(y^{2}\right)$ and $\mathcal{O}\left(\lambda^{2}\right)$ decay rates and scattering diagrams involving the (s)top. The expressions for the decay $D$ and (s)top scattering rates $S$ can be found in appendix A. The solutions to these Boltzmann 
equations are shown in Figs. 2,4, The baryon abundance is related to the (s)lepton abundance through

$$
Y_{B}=-\frac{8}{15} Y_{\Delta L_{\mathrm{tot}}}
$$

We have chosen $m_{\mathrm{eff}}=10^{-14} \mathrm{TeV}, M=10^{5} \mathrm{TeV}, M_{h}=125 \mathrm{GeV},|A|=1 \mathrm{TeV},|\lambda|=10^{-8}$, $\left|c_{1}\right|=0.001 \mathrm{TeV}$ and $m_{\Phi}=0.1 \mathrm{TeV} 1$ The evolution of the $Y_{B}$ abundance is similar to those found in [27, 39].

The DM abundances $Y_{\phi}$ and $Y_{\chi}$ are not sensitive to changes in the DM mass parameter $m_{\Phi}$ as long as it is a lot smaller than the heavy scale $M$ associated with the singlet (s)neutrino, $m_{\Phi} \ll M$. It is the coupling $\lambda$ and the positive mass dimension soft coupling $c_{1}$ that largely determine the order of magnitude in the DM abundances. With suitable choices of these couplings, the abundances $Y_{\chi}$ and $Y_{\phi}$ attain final values $\sim 10^{-11}$ suggesting that both the scalar and fermionic DM particles can be viable DM candidates.

At small couplings $\lambda \sim 10^{-8}$ and $c_{1} \sim 0.001 \mathrm{TeV}$, the asymmetric component is extremely tiny with abundance $\sim 10^{-17}$ and does not play a role in DM energy density. However, an increase in both DM sector couplings causes the magnitude of $Y_{\Delta \phi}$ to rise and also compels to consider scatterings where DM particles are destroyed and MSSM particles are produced. So, in order to have ADM as the primary source of all DM, it is crucial to find a balance between sufficient DM production through asymmetric decays and scattering processes

$$
\begin{aligned}
& \phi \phi \rightarrow \tilde{L}_{\alpha} H_{u}, \phi \phi \rightarrow L_{\alpha} \widetilde{H}_{u}^{c}, \\
& \chi \chi^{c} \rightarrow \tilde{L}_{\alpha} H_{u}, \chi \chi^{c} \rightarrow L_{\alpha} \widetilde{H}_{u}^{c}, \\
& \chi \phi \rightarrow L_{\alpha} H_{u}, \chi \phi \rightarrow \tilde{L}_{\alpha} \widetilde{H}_{u}^{c},
\end{aligned}
$$

where $\chi^{c}$ denotes the right-handed fermion obtained from $\chi$ through $\bar{\chi}^{\dot{\alpha}}=\epsilon^{\dot{\alpha} \dot{\beta}}\left(\chi_{\beta}\right)^{*}$, where $\epsilon^{\dot{\alpha} \dot{\beta}}=\left(-i \sigma^{2}\right)^{\dot{\alpha} \dot{\beta}}$. We believe it is possible to create conditions where the DM particles annihilate and the asymmetric component remains and forms a part of final DM abundance although this scenario entails a different parameter space in terms of DM couplings and other parameter inputs. We are studying this alternative in an upcoming paper.

We have explored the allowed parameter space and made scatter plots of the DM masses versus the DM coupling $|\lambda|$, Figs. 5-66. The fixed parameters are $m_{\mathrm{eff}}=10^{-14} \mathrm{TeV}, M=10^{5}$

\footnotetext{
${ }^{1}$ If the heavy (s)neutrinos are supposed to be produced thermally, large $M$ implies reheating temperature $T_{R}>M$. In many inflatory supergravity models this high $T_{R}$ leads to overproduction of gravitinos. However, recently it has been proposed that with the present value of the Higgs mass about $125 \mathrm{GeV}$ [42] the reheating temperature constraint could be relaxed, $T_{R} \sim 10^{9}-10^{10} \mathrm{GeV}$, not in conflict with our scenario [43.
} 
Table 1: The DM sector parameters and their scanned values.

\begin{tabular}{|c|c|}
\hline Parameters & Scanned values \\
\hline$|\lambda|$ & $10^{-10}-10^{-8}$ \\
\hline$\left|c_{1}\right|$ & $10^{-5}-10^{-3} \mathrm{TeV}$ \\
\hline$m_{\Phi}$ & $0.01-1 \mathrm{TeV}$ \\
\hline$m_{\text {Фsoft }}^{2}$ & $10^{-4}-1 \mathrm{TeV}^{2}$ \\
\hline
\end{tabular}

$\mathrm{TeV}$ and $A=1 \mathrm{TeV}$ while $|\lambda|,\left|c_{1}\right|, m_{\Phi}$ and $m_{\Phi \text { soft }}$ are varied as shown in Table 1 . The range for the soft parameter $\left|c_{1}\right|$ has been chosen so that $\left|c_{1}\right| \sim|\lambda| M$. The DM coupling $10^{-10}<|\lambda|<10^{-8}$ is the most favorable region of producing the correct DM abundance which is why this particular region is studied. The DM mass parameters are chosen to be in the weak scale and even in the $10 \mathrm{GeV}$ range which brings the typical ADM regime closer. The required DM abundances come from the relation

$$
m_{\chi}\left|Y_{\chi}\right|+m_{\phi}\left|Y_{\phi}\right| \lesssim \frac{3}{4} \frac{\Omega_{\mathrm{DM}}}{\Omega_{\gamma}} T
$$

and the baryon abundance has to satisfy

$$
\left|Y_{B}\right| \lesssim 8.8 \times 10^{-11}
$$

which we have used as constraints in extracting the allowed parameter space. Known parameter values from observations are $T=2.725 \mathrm{~K}, \Omega_{\mathrm{DM}}=0.11 / h^{2}$ and $\Omega_{\gamma}=2.47 \times 10^{-5} / h^{2}$. We have taken the lower limit in (3.9) to be $30 \%$ of the observed baryon abundance while in (3.8) the lower limit is $90 \%$ of the observed value. Figs. 5-6 reflect the hyperbola-like boundary set by Eq. (3.8), and there is an indication of slight preference of $|\lambda|$ values in the $\sim 10^{-9}$ ballpark. A similar observation of the favorable magnitude of $|\lambda|$ can be made from a plot with the left-hand side of (3.8) plotted against $|\lambda|$.

We have also determined the dependence of efficiency factors related to baryon and ADM production on the Yukawa and DM couplings. The efficiency factors $\eta_{B}$ and $\eta_{\mathrm{DM}}$ are shown in Figs. 7,8 and they are given by

$$
\eta_{B, \mathrm{DM}}=\left|\frac{Y_{\Delta L_{\mathrm{tot}}, \Delta \phi}}{2 \varepsilon_{L, \mathrm{DM}} Y_{\widetilde{N}}^{\mathrm{eq}}(z \approx 0)}\right| .
$$

The ADM production efficiency falls with rising $m_{\text {eff }}$ while with increasing $|\lambda| \eta_{\text {DM }}$ increases and after reaching a maximum a steep decline ensues. The fall in $\eta_{\mathrm{DM}}$ is due to the fact that 
while $|\lambda|$ increases, DM production climbs up but sooner or later washout processes overcome the production. This observation is also made in [38]. Of course a more accurate prediction of the DM efficiency factor can be made when the scenario better fulfills the criteria for the existence of ADM, which would mean larger DM couplings $|\lambda|$ (and $\left|c_{1}\right|$ ) and the inclusion of DM annihilations into MSSM particles.

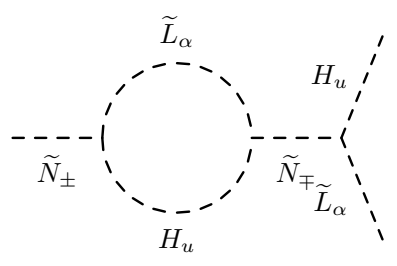

(a)

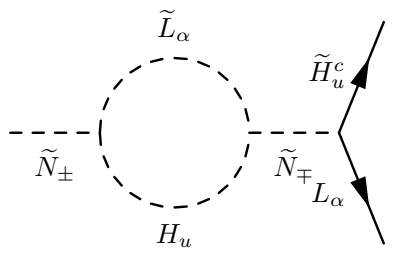

(c)

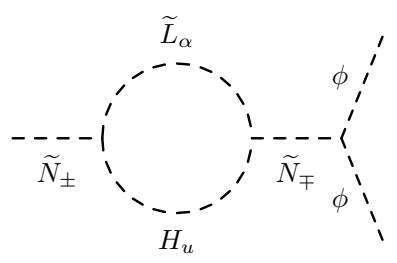

(e)

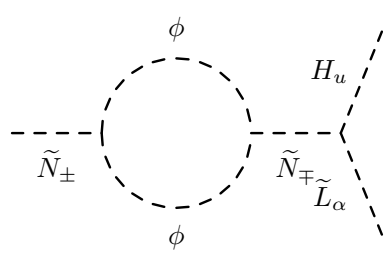

(b)

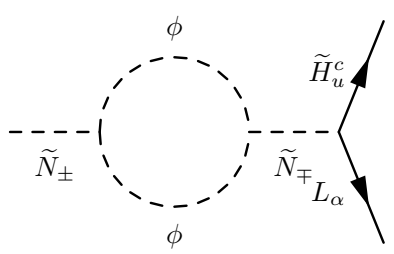

(d)

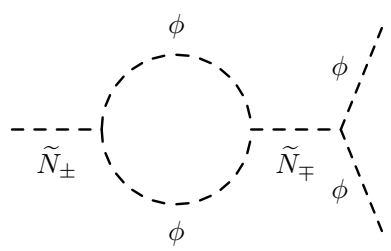

(f)

Figure 1: The relevant loop diagrams contributing to (s)lepton and DM asymmetries.

\section{Conclusions}

We have studied soft leptogenesis with an additional singlet DM superfield that couples to the singlet neutrino superfield. This framework allows the production of DM through the heavy (s)neutrino decays. In the weak coupling regime we have neglected suppressed higher order scattering processes, which does not make the ADM scenario viable due to the lack of DM annihilation channels. The absence of annihilations makes the DM produced in the tree level decays $\widetilde{N}_{ \pm} \rightarrow \chi \chi^{c}, \widetilde{N}_{ \pm} \rightarrow \phi \phi$ and $N \rightarrow \chi \phi$ stable.

It turns out that sufficient levels of baryons and DM can be produced in accordance with observations without the need for ADM. This is supported by the evolution of the final abun- 


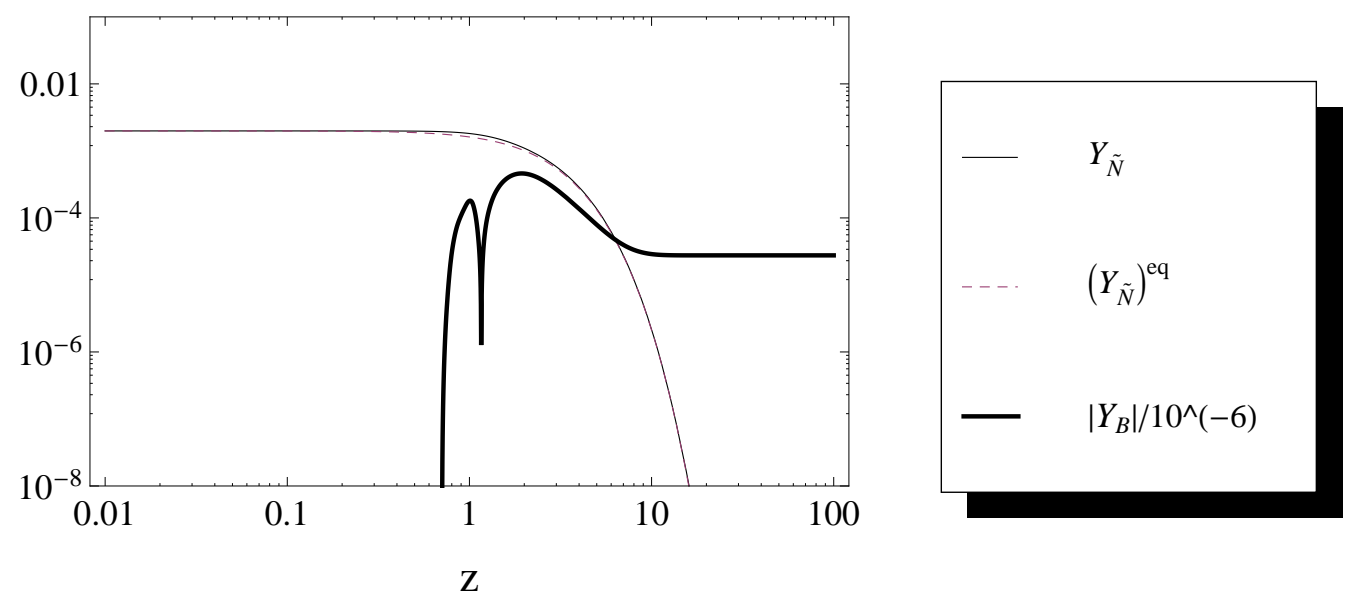

Figure 2: Baryon $\left|Y_{B}\right|$ and sneutrino $Y_{\widetilde{N}}$ abundances with $M=10^{5} \mathrm{TeV}, m_{\mathrm{eff}}=10^{-14} \mathrm{TeV}$, $A=1 \mathrm{TeV}, \lambda=10^{-8}$ and $m_{\Phi}=0.1 \mathrm{TeV}$.

dances $Y_{B}$ and $Y_{\chi, \phi}$ and parameter space related to the DM sector. We have produced scatter plots depicting the overall dependence of DM masses $m_{\chi, \phi}$ versus the DM coupling $\lambda$ with cosmological observations of the baryonic matter and DM energy densities as constraints, Eqs. (3.8) and (3.9) . The DM coupling $\lambda$ and its soft counterpart $c_{1}$ are independent from the MSSM and soft leptogenesis parameters and thus their magnitudes are fixed by the present DM abundance, and also the baryon abundance through loop effects in $\varepsilon_{L}$ in Eq. (2.7). The plots in Figs. 5 and 6 exhibit the fact that the observed DM energy density restricts the allowed region by making a hyperbola-like boundary.

For future studies, it would be interesting to see what is the correct parameter space that allows for ADM in this scenario. We believe this happens once we take into account the annihilation processes for the DM particles. The leading annihilation channel for scalar DM $\phi \phi \rightarrow \tilde{L}_{\alpha} H_{u}$ comes from the $F$-term potential. Other channels are mediated by $N$ and $\widetilde{N}_{ \pm}$. Thus, a stronger coupling of the DM sector can not only lead to higher levels of the asymmetric abundance $Y_{\Delta \phi}$ but also makes the DM annihilations significant which is a necessary precursor for the existence of ADM.

Acknowledgments The work of H.K. was supported by Alfred Kordelin Foundation. 

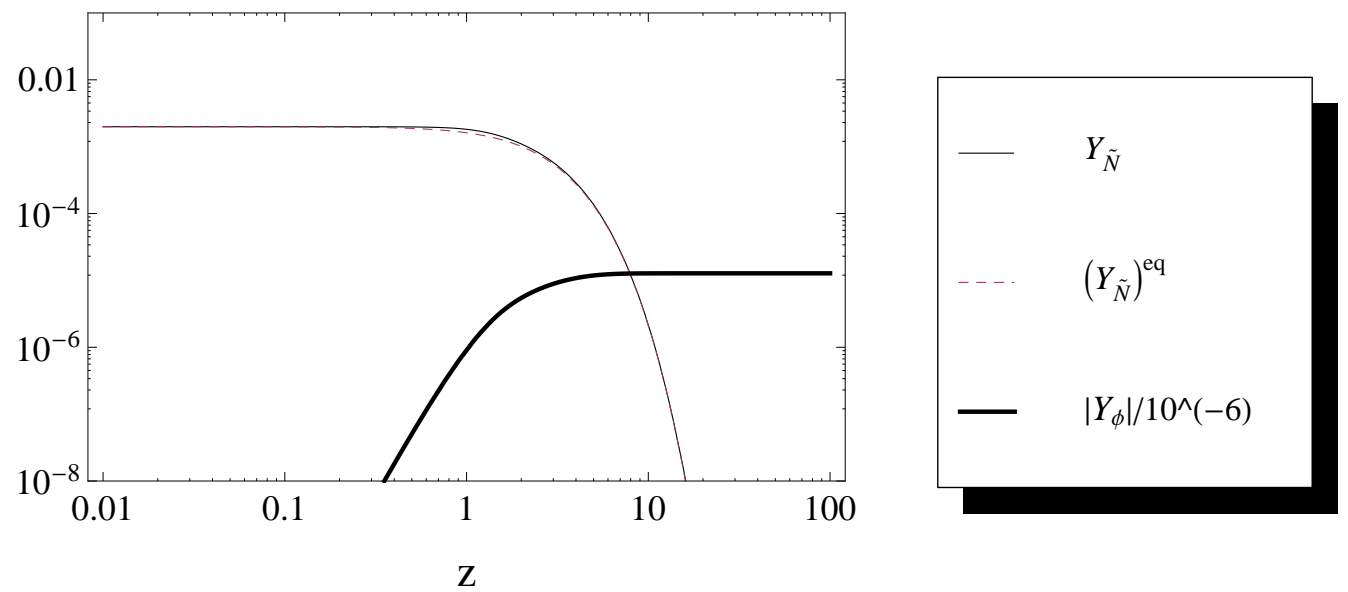

Figure 3: Scalar DM $\left|Y_{\phi}\right|$ and sneutrino $Y_{\widetilde{N}}$ abundances with $M=10^{5} \mathrm{TeV}, m_{\text {eff }}=10^{-14} \mathrm{TeV}$, $A=1 \mathrm{TeV},|\lambda|=10^{-8}, m_{\Phi}=0.1 \mathrm{TeV}$ and $\left|c_{1}\right|=0.001 \mathrm{TeV}$.

\section{A Decay and scattering rates}

The (s)top scattering rates are given by [40, 39, 41]

$$
\begin{aligned}
S_{t}^{(0)} & =\frac{K_{S}}{6} P_{0}(z), S_{t}^{(1)}=\frac{K_{S}}{6} P_{1}(z), \\
S_{t}^{(2)} & =\frac{K_{S}}{6} P_{2}(z), S_{t}^{(3)}=\frac{K_{S}}{6} P_{3}(z), \\
S_{t}^{(4)} & =\frac{K_{S}}{6} P_{4}(z), S_{t}^{(5)}=\frac{K_{S}}{6} P_{5}(z), \\
S_{t}^{(6)} & =\frac{K_{S}}{6} P_{6}(z), S_{t}^{(7)}=\frac{K_{S}}{6} P_{7}(z), \\
S_{t}^{(8)} & =\frac{K_{S S}}{6} P_{8}(z), S_{t}^{(9)}=\frac{K_{S S}}{6} P_{9}(z), \\
S_{22} & =3 \alpha_{t} \frac{M}{8 \pi^{2} H(z=1)} \frac{M}{v_{u}^{2}} \frac{m_{\mathrm{eff}}}{z} \frac{K_{1}(z)}{K_{2}(z)},
\end{aligned}
$$

where

$$
\begin{aligned}
K_{S} & =18 \alpha_{t} \frac{M}{v_{u}^{2}} m_{\mathrm{eff}} \frac{v_{u}^{2}}{M} \frac{3 \sqrt{5} M_{\mathrm{Pl}}}{16^{2} \pi^{2} \pi^{5 / 2} \sqrt{g_{*}} v_{u}^{2}}, \\
K_{S S} & =18 \alpha_{t} \frac{M}{v_{u}^{2}} m_{\mathrm{eff}} \frac{M^{2}+|A|^{2}}{M^{2}} \frac{v_{u}^{2}}{M} \frac{3 \sqrt{5} M_{\mathrm{Pl}}}{16^{2} \pi^{2} \pi^{5 / 2} \sqrt{g_{*}} v_{u}^{2}}
\end{aligned}
$$




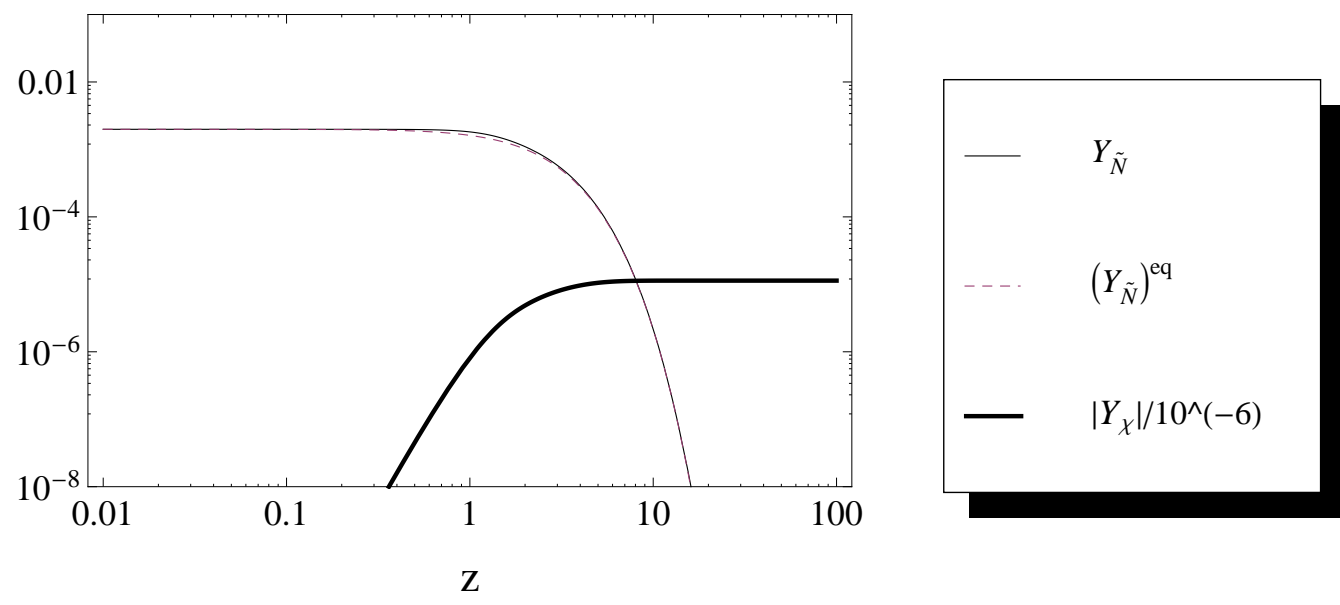

Figure 4: Fermionic DM $\left|Y_{\chi}\right|$ and sneutrino $Y_{\widetilde{N}}$ abundances with $M=10^{5} \mathrm{TeV}, m_{\mathrm{eff}}=10^{-14}$ $\mathrm{TeV}, A=1 \mathrm{TeV}, \lambda=10^{-8}, m_{\Phi}=0.1 \mathrm{TeV}$ and $\left|c_{1}\right|=0.001 \mathrm{TeV}$.

The reaction rates are found by integrating the functions $\left(x \equiv \psi / z^{2}\right)$

$$
\begin{aligned}
& f_{3}(x)=\frac{(x-1)^{2}}{x^{2}}, \\
& f_{4}(x)=\frac{x-1}{x}\left[\frac{x-2+2 a_{h}}{x-1+a_{h}}+\frac{1-2 a_{h}}{x-1} \log \left(\frac{x-1+a_{h}}{a_{h}}\right)\right], \\
& f_{0}(x)=\frac{1}{2} \frac{x^{2}-1}{x^{2}} \\
& f_{1}(x)=\frac{x-1}{x}\left[-\frac{2 x-1+2 a_{h}}{x-1+a_{h}}+\frac{x+2 a_{h}}{x-1} \log \left(\frac{x-1+a_{h}}{a_{h}}\right)\right], \\
& f_{2}(x)=\frac{x-1}{x}\left[-\frac{x-1}{x-1+2 a_{h}}+\log \left(\frac{x-1+a_{h}}{a_{h}}\right)\right], \\
& f_{5}(x)=\frac{1}{2} \frac{(x-1)^{2}}{x^{2}}, \\
& f_{6}(x)=\frac{x-1}{x}\left[-2+\frac{x-1+2 a_{h}}{x-1} \log \left(\frac{x-1+a_{h}}{a_{h}}\right)\right], \\
& f_{7}(x)=-\frac{x-1}{x-1+2 a_{h}}+\log \left(\frac{x-1+a_{h}}{a_{h}}\right), \\
& f_{8}(x)=\frac{x-1}{x^{2}}, \\
& f_{9}(x)=\frac{1}{x}\left[-\frac{x-1}{x-1+a_{h}}+\log \left(\frac{x-1+a_{h}}{a_{h}}\right)\right],
\end{aligned}
$$




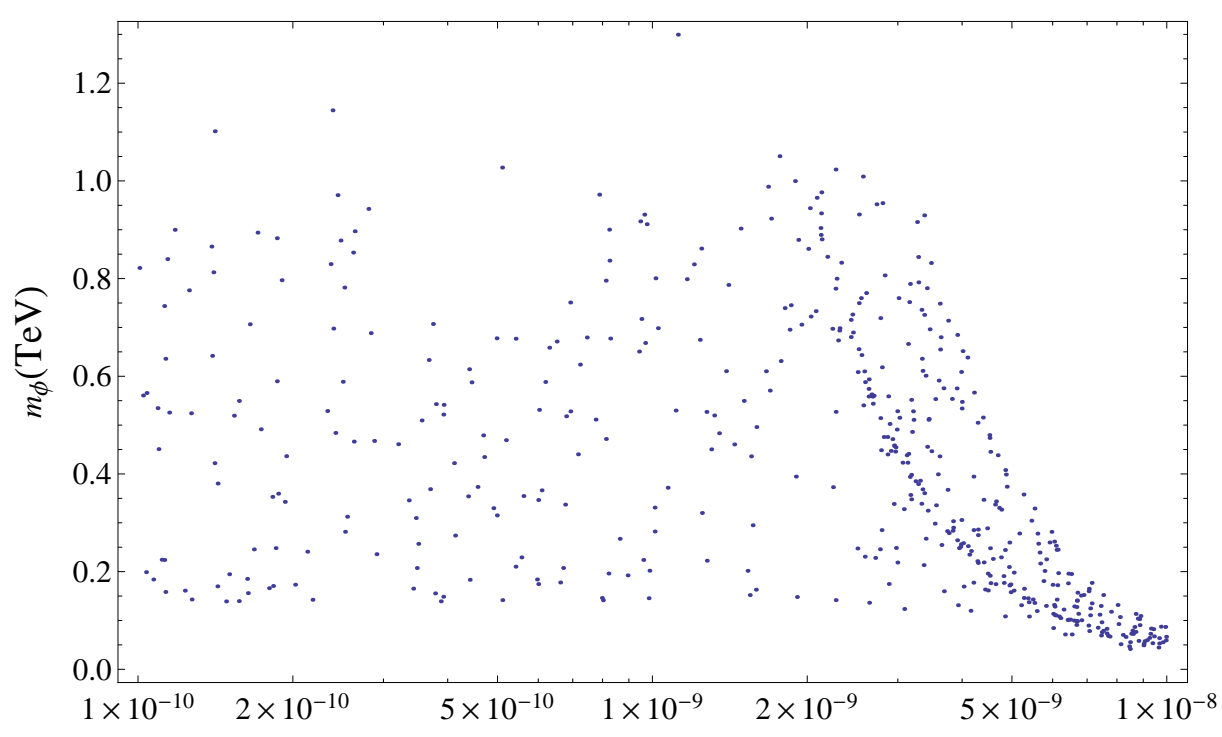

$|\lambda|$

Figure 5: Scatter plot of $m_{\phi}$ vs $|\lambda|$ with the present baryon and DM abundances as constraints, Eqs. (3.8) and (3.9).

where $a_{h}=\left(M_{h} / M\right)^{2}$ with $M_{h}$ standing for the mass of the Higgs boson. The integrals determining the reaction rates are of the form

$$
P_{i}(z)=\frac{K_{2}(z)}{z^{2}} \int_{z^{2}}^{\infty} d \psi f_{i}(\psi) \sqrt{\psi} K_{1}(\sqrt{\psi}) .
$$

The (s)neutrino decay rates are given by

$$
\begin{aligned}
D_{N} & =\frac{M}{v_{u}^{2}} m_{\mathrm{eff}} \frac{M}{(4 \pi)} z \frac{K_{1}(z)}{K_{2}(z)}, \\
D_{\widetilde{N}} & =\left(\frac{1}{8 \pi M}\left(M^{2}+|A|^{2}\right)+\frac{1}{8 \pi} M\right) \frac{M}{v_{u}^{2}} m_{\mathrm{eff}} z \frac{K_{1}(z)}{K_{2}(z)} \frac{1}{H(z=1)}, \\
D_{\widetilde{N} \phi \phi} & =\frac{1}{4 \pi M}\left(\frac{\left|c_{1}\right|^{2}}{2}+\frac{M^{2}|\lambda|^{2}}{8}\right) z \frac{K_{1}(z)}{K_{2}(z)} \frac{1}{H(z=1)}, \\
D_{\widetilde{N} \chi \chi} & =\frac{1}{8 \pi} M|\lambda|^{2} z \frac{K_{1}(z)}{K_{2}(z)} \frac{1}{H(z=1)}, \\
D_{\widetilde{N} \chi \phi} & =\frac{1}{8 \pi} M|\lambda|^{2} z \frac{K_{1}(z)}{K_{2}(z)} \frac{1}{H(z=1)}, \\
D_{\widetilde{N}}^{(3)} & =\frac{3 \alpha_{t}}{64 \pi^{2}} M \frac{M}{v_{u}^{2}} \frac{m_{\mathrm{eff}}}{H(z=1)} z \frac{K_{1}(z)}{K_{2}(z)} .
\end{aligned}
$$




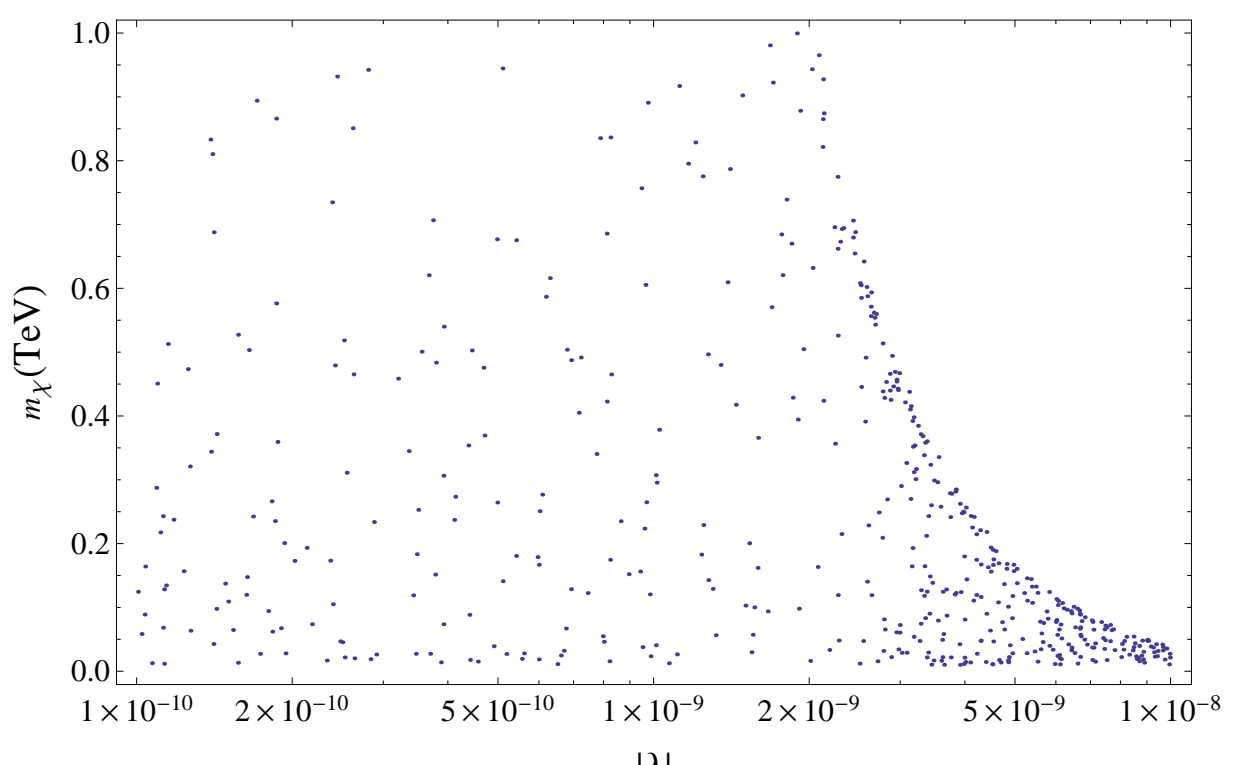

$|\lambda|$

Figure 6: Scatter plot of $m_{\chi}$ vs $|\lambda|$ with the present baryon and DM abundances as constraints, Eqs. (3.8) and (3.9).

\section{References}

[1] WMAP Collaboration E. Komatsu et al., Seven-Year Wilkinson Microwave Anisotropy Probe (WMAP) Observations: Cosmological Interpretation, Astrophys. J. Suppl. 192, 18 (2011).

[2] M. Fukugita and T. Yanagida, Baryogenesis Without Grand Unification, Phys. Lett. B 174:45 (1986).

[3] H. Goldberg Constraint on the Photino Mass from Cosmology, Phys. Rev. Lett. 50 , 1419 (1983); Supersymmetric relics from the big bang. Ellis, J. Hagelin, D. V. Nanopoulos, K. Olive, M. Srednicki, Nucl. Phys. B 238, 453 (1984)

[4] F. D. Steffen, Dark-matter candidates, Eur. Phys. J. C59 (2009) 557-588; M. Kawasaki, K. Kohri, T. Moroi, A. Yotsuyanagi, Big-bang nucleosynthesis and gravitinos, Phys. Rev. D78 (2008) 065011.

[5] S. Nussinov, Technocosmology - could a technibaryon excess provide a "natural" missing mass candidate?, Phys. Lett. B 165 (1985) 55; S. M. Barr, Baryogenesis, sphalerons, and the cogeneration of dark matter, Phys. Rev. D 44, 3062-3066 (1991); S. M. Barr, R. S. Chivukula and E. Farhi, Electroweak fermion number violation and the production of 


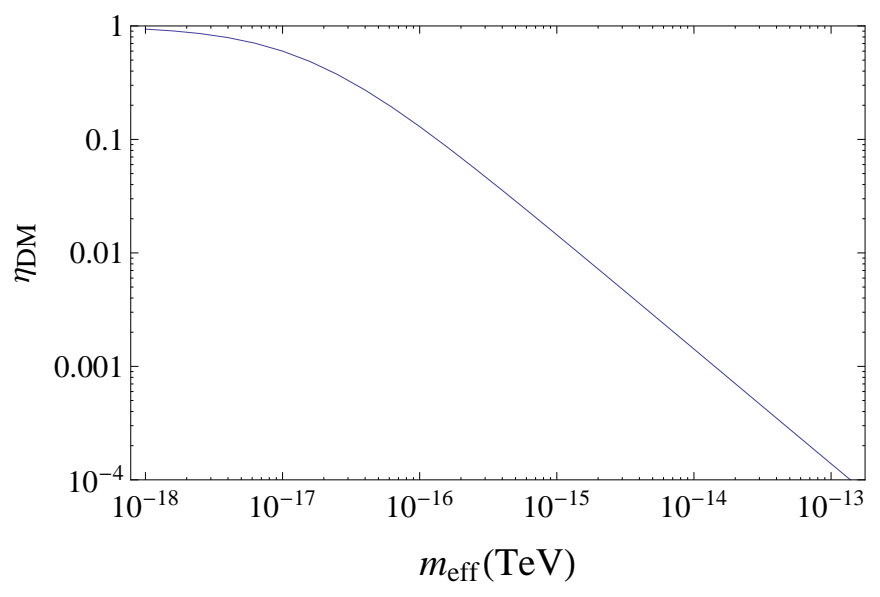

Figure 7: The efficiency factor $\eta_{\mathrm{DM}}$ for asymmetric DM production vs $m_{\mathrm{eff}}$.

stable particles in the early universe, Phys. Lett. B 241 (1990) 387-391; D. B. Kaplan, Single explanation for both baryon and dark matter densities, Phys. Rev. Lett. 68 (1992) 741; D. E. Kaplan, M. A. Luty and K. M. Zurek, Asymmetric dark matter, Phys Rev. D 79, 115016 (2009); T. Cohen, and K. M. Zurek, Leptophilic Dark Matter from the Lepton Asymmetry, Phys. Rev. Lett. 104, 101301 (2010); T. Cohen, D. J. Phalen, A. Pierce and K. M. Zurek, Asymmetric dark matter from a GeV hidden sector, Phys. Rev. D 82, 056001 (2010); G. R. Farrar and G. Zaharijas, Dark Matter and the Baryon Asymmetry of the Universe, Phys. Rev. Lett. 96, 041302 (2006); D. Hooper, J. March-Russell and S. M. West, Asymmetric sneutrino dark matter and the $\Omega_{b} / \Omega_{D M}$ puzzle, Phys. Lett. B 605 (2005) 228-236; R. Kitano and I. Low, Dark matter from baryon asymmetry, Phys. Rev. D 71 (2005) 023510; K. Agashe and G. Servant, Baryon number in warped grand unified theories: model building and (dark matter related) phenomenology, JCAP 0502 (2005) 002; R. Kitano, H. Murayama and M. Ratz, Unified origin of baryons and dark matter, Phys. Lett. B 669 (2008) 145-149; E. Nardi, F. Sannino and A. Strumia, Decaying dark matter can explain the $e^{ \pm}$excesses, JCAP 0901 (2009) 043; J. Shelton and K. M. Zurek, Darkogenesis: A baryon asymmetry from the dark matter sector, Phys. Rev. D 82 (2010) 123512; H. Davoudiasl, D. E. Morrissey, K. Sigurdson and S. Tulin, Unified Origin for Baryonic Visible Matter and Antibaryonic Dark Matter, Phys. Rev. Lett. 105 (2010) 211304; N. Haba and S. Matsumoto, Baryogenesis from Dark Sector, Prog. Theor. Phys. 125 (2011) 1311-1316; P.-H. Gu, M. Lindner, U. Sarkar and X. Zhang, Weakly interacting dark matter and baryogenesis, Phys. Rev. D 83 (2011) 055008; J. McDonald, Relating the baryon asymmetry to the thermal relic dark matter density, Phys. Rev. D 83 


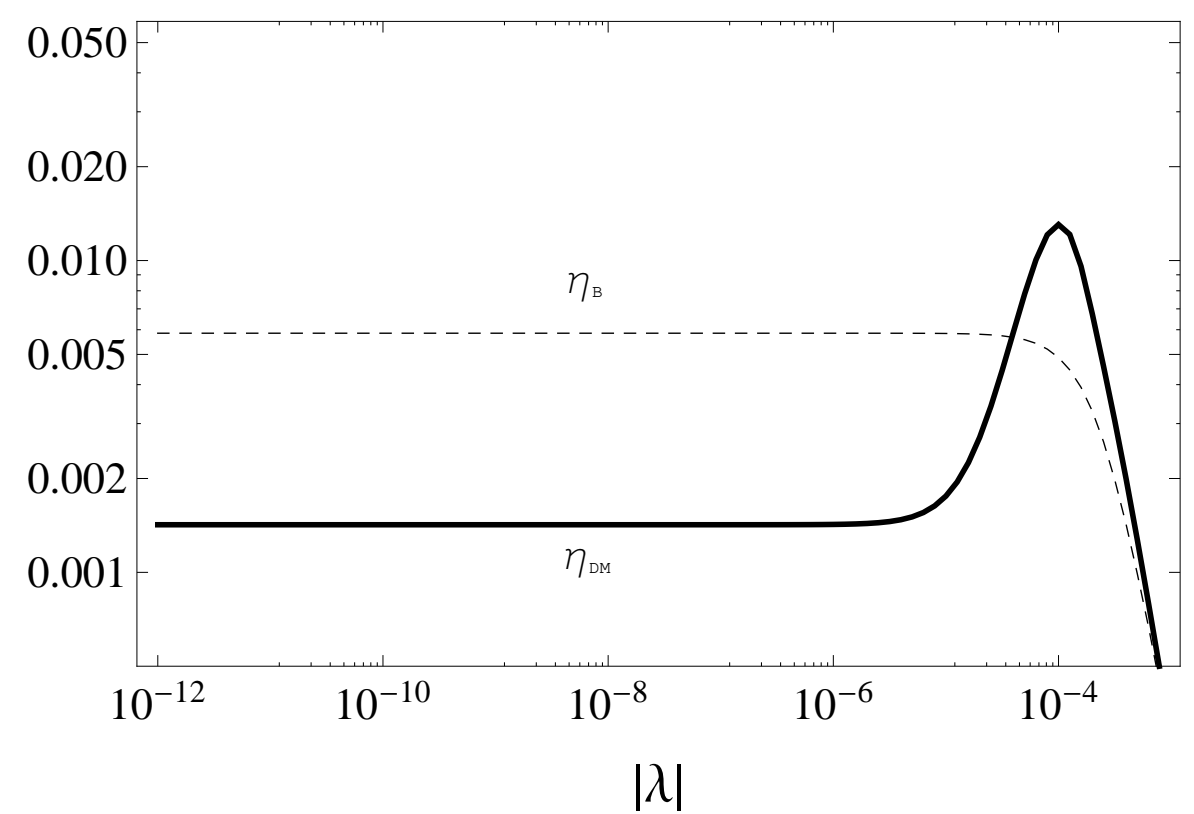

Figure 8: The efficiency factors $\eta_{B}$ and $\eta_{\mathrm{DM}}$ vs $|\lambda|$ for $m_{\mathrm{eff}}=10^{-14} \mathrm{TeV}$.

(2011) 083509; C. Cheung, G. Elor, L. J. Hall and P. Kumar, Origins of hidden sector dark matter I: cosmology, JHEP 1103 (2011) 042; C. Cheung, G. Elor, L. J. Hall and P. Kumar, Origins of hidden sector dark matter II: collider physics, JHEP 1103 (2011) 085; L. J. Hall, J. March-Russell and S. M. West, A Unified Theory of Matter Genesis: Asymmetric Freeze-In, arXiv:1010.0245; B. Dutta and J. Kumar, Asymmetric dark matter from hidden sector baryogenesis, Phys. Lett. B 699 (2011) 364-367; B. Feldstein and A. L. Fitzpatrick, Discovering asymmetric dark matter with anti-neutrinos, JCAP 1009 (2010) 005; M. R. Buckley and Lisa Randall, Xogenesis, JHEP 1109 (2011) 009; H. Davoudiasl, D. E. Morrissey, K. Sigurdson and S. Tulin, Baryon destruction by asymmetric dark matter, Phys. Rev. D 84, 096008 (2011); N. F. Bell, K. Petraki, I. M. Shoemaker and A. R. Volkas, Dark and visible matter in a baryon-symmetric universe via the Affleck-Dine mechanism, Phys. Rev. D 84, 123505 (2011); D. E. Kaplan, G. Z. Krnjaic, K. R. Rehermann and C. M. Wells, Dark atoms: asymmetry and direct detection, JCAP 1110 (2011) 011; C. Cheung and K. M. Zurek, Affleck-Dine cogenesis, Phys. Rev. D 84, 035007 (2011); Y. Cui, L. Randall and B. Shuve, Emergent dark matter, baryon, and lepton numbers, JHEP08 (2011) 073; M. L. Graesser, I. M. Shoemaker and L. Vecchi, Asymmetric WIMP dark matter, JHEP10 (2011) 110; S. M. Barr, Unification and cogeneration of dark matter and baryonic matter, Phys. Rev. D 85, 013001 (2012); M. R. Buckley and S. Profumo, 
Regenerating a Symmetry in Asymmetric Dark Matter, Phys. Rev. Lett. 108, 011301 (2012); J. March-Russell and M. McCullough, Asymmetric dark matter via spontaneous co-genesis, JCAP 1203 (2012) 019; T. Lin, H.-B. Yu and K. M. Zurek, Symmetric and asymmetric light dark matter, Phys. Rev. D 85, 063503 (2012); M. Cirelli, P. Panci, G. Servant and G. Zaharijas, Consequences of DM/antiDM oscillations for asymmetric WIMP dark matter, JCAP03 (2012) 015; M. Blennow, E. Fernandez Martinez, O. Mena, J. Redondo and P. Serra, Asymmetric Dark Matter and Dark Radiation, JCAP07 (2012) 022; Z. Kang, J. Li, T. Li, T. Liu and J. Yang, Asymmetric Sneutrino Dark Matter in the NMSSM with Minimal Inverse Seesaw, arXiv:1102.5644; J. J. Heckman and S.-J. Rey, Baryon and dark matter genesis from strongly coupled strings, JHEP 1106 (2011) 120; K. Kohri, A. Mazumdar, N. Sahu and P. Stephens, Probing the unified origin of dark matter and baryon asymmetry at PAMELA and Fermi Large Area Telescope, Phys. Rev. D 80, 061302(R) (2009).

[6] M. Flanz, E. A. Paschos and U. Sarkar, Baryogenesis from a lepton asymmetric universe, Phys. Lett. B345:248-252 (1995).

[7] L. Covi, E. Roulet and F. Vissani, Cp violating decays in leptogenesis scenarios, Phys. Lett. B384:169-174 (1996).

[8] M. Plümacher, Baryogenesis and lepton number violation, Z. Phys. C74:549-559 (1997).

[9] W. Buchmüller and M. Plümacher, CP asymmetry in Majorana neutrino decays, Phys. Lett. B431:354-362 (1998).

[10] W. Buchmüller, P. Di Bari and M. Plümacher, Cosmic microwave background, matterantimatter asymmetry and neutrino masses, Nucl. Phys. B643:367-390 (2002).

[11] R. Barbieri, P. Creminelli, A. Strumia and N. Tetradis, Baryogenesis through leptogenesis, Nucl. Phys. B575:61-77 (2000).

[12] G. F. Giudice, A. Notari, M. Raidal, A. Riotto and A. Strumia, Towards a complete theory of thermal leptogenesis in the SM and MSSM, Nucl. Phys. B685:89-149 (2004).

[13] A. Pilaftsis and T. E. J. Underwood, Electroweak-scale resonant leptogenesis, Phys. Rev. D72, 113001 (2005).

[14] E. Nardi, Y. Nir, E. Roulet and J. Racker, The importance of flavor in leptogenesis, JHEP 0601 (2006) 164. 
[15] A. Abada, S. Davidson, F. Josse-Michaux, M. Losada and Antonio Riotto, Flavour issues in leptogenesis, JCAP 0604 (2006) 004.

[16] A. Abada, S. Davidson, A. Ibarra, F. Josse-Michaux, M. Losada and A. Riotto, Flavour matters in leptogenesis, JHEP 0609 (2006) 010.

[17] S. Blanchet and P. Di Bari, Flavor effects on leptogenesis predictions, JCAP 0703 (2007) 018.

[18] M. Flanz, E. A. Paschos, U. Sarkar and J. Weiss, Baryogenesis through mixing of heavy Majorana neutrinos, Phys. Lett. B389:693-699 (1996).

[19] L. Covi and E. Roulet, Baryogenesis from mixed particle decays, Phys. Lett. B399:113-118 (1997).

[20] A. Pilaftsis, CP violation and baryogenesis due to heavy Majorana neutrinos, Phys. Rev. D56:5431-5451 (1997); A. Pilaftsis, Heavy Majorana neutrinos and baryogenesis, Int. J. Mod. Phys. A14:1811-1857 (1999).

[21] A. Pilaftsis and T. E. J. Underwood, Resonant leptogenesis, Nucl. Phys. B692:303-345 (2004).

[22] A. Anisimov, A. Broncano and M. Plümacher, The CP-asymmetry in resonant leptogenesis, Nucl. Phys. B737:176-189 (2006).

[23] R. Allahverdi, B. Dutta and A. Mazumdar, Sleptogenesis, Phys. Rev. D 67, 123515 (2003).

[24] P. Minkowski, mu-> e gamma at a Rate of One Out of 1-Billion Muon Decays?, Phys. Lett. B67:421 (1977); M. Gell-Mann, P. Ramond and R. Slansky in: Supergravity, P. van Nieuwenhuizen, D. Z. Freedman (Eds.), North-Holland, Amsterdam, p. 315 (1979); T. Yanagida, in: Proceedings of the Workshop on the Baryon Number of the Universe and Unified Theories, Tsukuba, Japan 13-14 (1979); S. L. Glashow, NATO Adv. Study Inst. Ser. B Phys. 59:687 (1979).

[25] R. N. Mohapatra and G. Senjanovic, Neutrino Mass and Spontaneous Parity Violation, Phys. Rev. Lett. 44:912-915 (1980).

[26] Y. Grossman, T. Kashti, Y. Nir and E. Roulet, Leptogenesis from Supersymmetry Breaking, Phys. Rev. Lett. 91, 251801 (2003). 
[27] G. D'Ambrosio, G. F. Giudice and M. Raidal, Soft leptogenesis, Phys. Lett. B575:75-84 (2003).

[28] L. Boubekeur, Leptogenesis at low scale, hep-ph/0208003.

[29] N. Cosme, L. Lopez Honorez and M. H. G. Tytgat, Are leptogenesis and dark matter related?, Phys. Rev. D 72 (2005) 043505.

[30] P.-H. Gu, U. Sarkar and X. Zhang, Visible and dark matter genesis and cosmic positron and electron excesses, Phys. Rev. D 80 (2009) 076003.

[31] P.-H. Gu and U. Sarkar, Common origin of visible and dark universe, Phys. Rev. D 81 (2010) 033001.

[32] H. An, S.-L. Chen, R. N. Mohapatra and Y. Zhang, Leptogenesis as a common origin for matter and dark matter, JHEP 03 (2010) 124.

[33] M. Blennow, B. Dasgupta, E. Fernandez-Martinez and N. Rius, Aidnogenesis via leptogenesis and dark sphalerons, JHEP 1103 (2011) 014.

[34] A. Falkowski, J. T. Ruderman and T. Volansky, Asymmetric dark matter from leptogenesis, JHEP 1105 (2011) 106.

[35] C. Arina and N. Sahu, Asymmetric inelastic inert doublet dark matter from triplet scalar leptogenesis, Nucl. Phys. B 854 (2012) 666-699.

[36] C. Arina, J.-O. Gong and N. Sahu, Unifying darko-lepto-genesis with scalar triplet inflation, Nucl. Phys. B 865 (2012) 430-460.

[37] E.-J. Chun, Leptogenesis origin of Dirac gaugino dark matter, Phys. Rev. D 83 (2011) 053004 .

[38] E.-J. Chun, Minimal dark matter and leptogenesis, JHEP 1103 (2011) 098.

[39] C. S. Fong, M. C. Gonzalez-Garcia and E. Nardi, Leptogenesis from Soft Supersymmetry Breaking: Soft Leptogenesis, Int. J. Mod. Phys. A 26 (2011) 3491-3604.

[40] W. Buchmüller, P. Di Bari and M. Plümacher, Leptogenesis for pedestrians, Ann. Phys. 315:305-351 (2005).

[41] M. Plümacher, Baryon asymmetry, neutrino mixing and supersymmetric SO(10) unification, Nucl. Phys. B 530 (1998) 207-246. 
[42] G. Aad et al. [The ATLAS Collaboration], Observation of a new particle in the search for the Standard Model Higgs boson with the ATLAS detector at the LHC, Phys. Lett. B 716 (2012) 1-29; S. Chatrchyan et al. [The CMS Collaboration], Observation of a new boson at a mass of $125 \mathrm{GeV}$ with the CMS experiment at the LHC, Phys. Lett. B 716 (2012) 30-61.

[43] K. Nakayama, F. Takahashi and T. T. Yanagida, Eluding the Gravitino Overproduction in Inflaton Decay, Phys. Lett. B 718 (2012) 526-531. 